\title{
Control de temperatura en intercambiadores de calor tipo coraza-tubo: una revisión realizada a la industria ${ }^{1}$
}

\section{Temperature control in shell-tube heat exchangers: a review of the industry}

\author{
J. G. Álvarez, J. M. Armero and C. A. Urrutia \\ Recibido: junio 13 de 2019 - Aceptado: junio 20 de 2020
}

\begin{abstract}
Resumen- Esta revisión sobre lazos de temperatura en intercambiadores de calor tipo coraza-tubo, parte de un estudio realizado a la industria de la región del Valle del Cauca donde se identifican en la ingeniería de control de los intercambiadores de calor las siguientes deficiencias: desconocimiento del modelo, inexperiencia en la selección de las estrategias de control, pobre sintonía de los algoritmos de control y desconocimiento de criterios de evaluación de desempeño. La literatura consultada muestra una gran cantidad de métodos de identificaciones de modelos y estrategias de controles y sintonías aplicados a intercambiadores, sin embargo, en su mayoría son implementados a nivel de simulación, despreciando las no linealidades e interacciones del intercambiador en el proceso industrial. La revisión presenta diferentes métodos y conceptos usados para la obtención de modelos, estrategias de control, sintonía y evaluación de lazos de control que se encuentran en la literatura desde el 2010.
\end{abstract}

Palabras Clave - Control de temperatura, control de procesos industriales, controladores PID, criterios de desempeño, diseño de control, sistemas con retardo, sistemas de control cerrado.

Abstract - This review on temperature control loops in shell-andtube heat exchangers is part of a study conducted to the industry in the region of Valle del Cauca where the following deficiencies

\footnotetext{
${ }^{1}$ Producto derivado del proyecto de investigación aplicada denominado "Valoración de metodologías de sintonía de controladores PID, para implementar mejoras en el control de plantas de procesos continuos", apoyado por el Servicio Nacional de Aprendizaje del SENA a través del programa de Investigación e Innovación en el SENA - SENNOVA

J. G. Álvarez, SENA, Cali, Colombia, email: jgalvarez@sena.edu.co.

J. M. Armero, SENA, Cali, Colombia, email: juan.armero@ correounivalle.edu.co.

C. A. Urrutia, SENA, Cali, Colombia, email: caurrutia@ sena.edu.co.
}

Como citar este artículo: Álvarez, J. G., Armero, J. M., y Urrutia, C. A. Control de temperatura en intercambiadores de calor tipo coraza-tubo: una revisión realizada a la industria, vol. 14, no. 27, pp. 41-49, enero-junio 2020. DOI: https://doi.org/10.31908/19098367.1763.

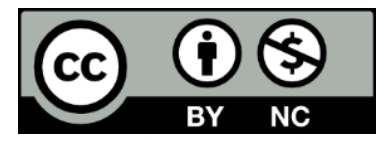

Attribution-NonCommercial 4.0 Intenational (CC By-NC 4.0) were identified in the control engineering of heat exchangers: lack of knowledge of the model, inexperience in the selection of control strategies, poor tuning of control algorithms and lack of knowledge of performance evaluation criteria. The literature consulted shows a great number of methods of model identification and control strategies and tuning applied to heat exchangers, however, most of them are implemented at the simulation level, disregarding the non-linearities and interactions of the exchanger in the industrial process. The review presents different methods and concepts used to obtain models, control strategies, tuning and evaluation of control loops that are in the literature since 2010.

Keywords - Temperature control, industrial process control, PID controller, system performance, control design, delay systems, Closed loop systems.

\section{INTRODUCCIÓN}

E n el sector industrial uno de los procesos importantes es la transferencia de calor, por ejemplo, la refrigeración, pasteurización y esterilización. La industria aprovecha las propiedades de la transferencia térmica utilizando dispositivos diseñados para elevar o disminuir la temperatura de materia prima o producto, denominando a esta técnica "intercambio de calor". Existen varios tipos de intercambiadores de los cuales el intercambiador de coraza tubo es el más común (sobre un $30 \%$ de los intercambiadores instalados) seguido por el de platos $(20 \%)$ [1].

La industria del Valle del Cauca (sector químico, papelero, alimenticio, farmacéutico) emplea intercambiadores de calor para suplir los servicios auxiliares de vapor y/o agua caliente. Con la finalidad de cumplir con estándares de producción y seguridad de las plantas, se han automatizado los procesos donde intervienen el intercambiador de calor, incorporando estrategias de control que regulan la temperatura de salida del producto a valores deseados. Sin embargo, el desconocimiento de la teoría de control por parte de los operarios y técnicos encargados de las plantas ocasiona estados de ineficiencia y aumentos de costos en la operación de los sistemas de intercambio térmico. Con base a entrevistas y visitas técnicas a empresas de la región, se detectó que en el $100 \%$ de las empresas encuestadas, la sintonía de los controladores de los intercambiadores de calor se realiza a partir de pruebas 
empíricas de ensayo y error basadas en la experiencia, impactando la calidad del producto final. Este panorama no es un problema propio de la región del Valle del Cauca, en el 2003 [2] se realizó observaciones en lazos de control de industrias Norte Americanas detectando que el $85 \%$ de los controladores presentaban una pobre sintonía y el $30 \%$ operaba en modo manual. Entre los años 2004 y 2005, en [3] se realizó un estudio similar, mostrando los resultados de una auditoría a 700 lazos de control en 12 compañías brasileras, estableciendo las siguientes problemáticas: válvulas con excesivo desgaste (14\% de los casos), válvulas con problemas de adherencia e histéresis (15\%), lazos operando en modo manual (16\%), controladores saturados (24\%) y oscilaciones con problemas de sintonía, acoplamiento, disturbios y fallos de actuadores $(41 \%)$.

Lo anterior evidencia la necesidad de fortalecer los métodos y procedimientos aplicados en la industria en torno a la sintonía de lazos de control, con el propósito de brindar solución a muchas de estas problemáticas y mejorar la productividad en la operación. El fortalecimiento del conocimiento debe realizarse en todas las etapas de la ingeniería de control [4]: ingeniería conceptual, ingeniería básica, ingeniería de detalle, implementación, puesta en marcha y mantenimiento.

En la ingeniería conceptual se analiza y se caracteriza el proceso. En el caso del intercambiador de calor, sus características no lineales y disturbios, con rangos de temperaturas de productos amplios y variables, representa un sistema difícil de controlar. Aunque el PID es el controlador más simple y comúnmente usado para el control de intercambiadores, el $62 \%$ de las empresas encuestadas lo emplean, no siendo apropiado para un proceso de características no lineales o tiempos muertos variables [5]. Considerando lo anterior, existen un gran número de algoritmos de control implementados en intercambiadores de calor: desde el tradicional PID [6] hasta controles inteligentes [7]-[10], robustos [11]-[13] y no lineales [5].

La ingeniería básica establece los requerimientos o especificaciones de diseño y define la estrategia de control. Las estrategias de control más empleadas a nivel académico y a nivel industrial son: el control realimentado [14] con algoritmos de control de PI, PID, PID avanzado y PID adaptativo; el control anticipativo, usado en conjunto con el realimentado para aumentar los grados de control del sistema; la estrategia de control cascada usada para dar robustez y velocidad de respuesta; control por derivación [15] y el control manual, empleado en aquellos casos en el que no se requiere un control preciso y robusto de la temperatura. La Fig. 1, visualiza los resultados de las visitas a la industria del Valle del Cauca.

La ingeniería de detalle implica obtener el modelo matemático del proceso y los parámetros de sintonía del controlador. Obtener el modelo matemático, es una fase fundamental en el diseño de los sistemas de control, ya que afecta directamente el desempeño y robustez de los controladores.

Los métodos de identificación que han sido aplicados en intercambiadores de calor a nivel de investigación, con potencial para ser aplicados a nivel industrial son los métodos no paramétricos, paramétricos y el analíticos [16], [17]. Sin embargo, en el $100 \%$ de las empresas consultadas, afirmaron que el modelo no es necesario a la hora de implementar una estrategia de control clásico debido a que la sintonía del controlador PID lo realizan de manera heurística. Las empresas no conocen el modelo de su proceso, por dos razones: falta de conocimiento de teoría de identificación de sistemas o por el arduo trabajo que implica esta tarea.

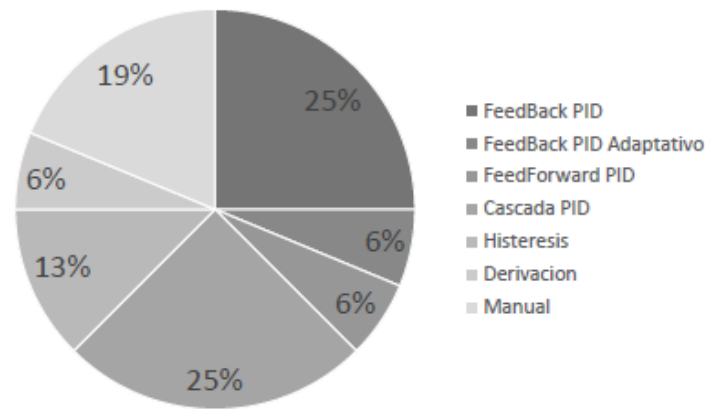

Fig. 1. Estrategias de control de la industria detectadas en la encuesta realizada por el grupo de institución UIADTI - Sena en la industria Vallecaucana.

La sintonía del sistema de control hace referencia al ajuste de los parámetros del controlador para obtener una respuesta "optima" o deseada del intercambiador de calor. Siendo una respuesta "optima" para el proceso según las especificaciones de diseño, por ejemplo: estabilidad, regulación, rechazo de disturbios, tiempo de respuesta, oscilaciones, error, eficiencia energética; siendo estas especificaciones las bases para definir los índices de desempeño para la evaluación del lazo de control en la ingeniería de puesta en marcha. Esta revisión de control de temperatura en intercambiadores de calor tipo coraza tubo aborda las principales estrategias de control empleadas en la industria y para investigación, técnicas para la obtención de modelo, algoritmos de sintonía de controladores y los índices de evaluación de desempeño de los lazos de control de temperatura.

\section{CONTROL DE TEMPERATURA - INTERCAMBIADOR DE CALOR}

Los intercambiadores de calor facilitan la transferencia térmica entre dos fluidos que se encuentran a distintas temperaturas, generalmente evitando la mezcla entre sí. El proceso de transferir el calor de un medio a otro en el intercambiador se lleva a cabo mediante los fenómenos conocidos como conducción y convección [18]. Por conducción, se entiende como la transferencia de energía de las partículas más energéticas de una substancia hacia las adyacentes menos energéticas, como resultado de interacciones entre esas partículas. La convección se da entre una superficie sólida y el líquido o gas adyacente que está en movimiento; cuanto mayor es la velocidad del fluido, mayor es la transferencia por convección.

Existe una gran variedad de intercambiadores térmicos, por ejemplo [19]: torres de enfriamiento (contacto directo), tubos concéntricos, tubos coraza, placas; siendo el de tubo-coraza (Fig. 2) el más utilizado en aplicaciones a nivel industrial dado que permite temperaturas y presiones superiores a los de placas $\left(300\right.$ psia y $\left.150^{\circ} \mathrm{C}\right)$. 


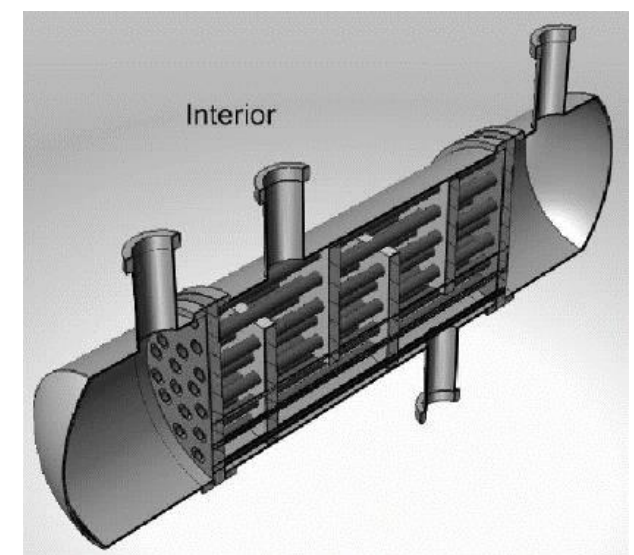

Fig. 2. Intercambiador de calor coraza-tubo.

El intercambiador de placas está orientado a aplicaciones con sustancias inocuas y el de tubo-coraza a aplicaciones de la industria en general. De acuerdo con la función en el proceso, los intercambiadores de calor se pueden clasificar en:

- Enfriador de líquidos o gases por medio de agua.

- Permutador, realiza una doble función, calentar y enfriar los dos fluidos que intervienen en la operación.

- Calentador, imparte calor a un fluido por medio de vapor.

- Rehervidor, buscar rehervir el líquido necesario para la destilación.

- Condensador, condensa vapor o mezcla de vapores.

- Vaporizador, es un calentador que vaporiza parte de un fluido.

- Caldera de recuperación, genera vapor a partir de gases de proceso.

Dado que el intercambiador de calor es usado en la industria alimentaria, petroquímica, farmacéutica, la industria de pulpa y papel, servicios auxiliares industriales de calentamiento de agua, generación de vapor, recuperadores, enfriadores; se hace indispensable realizar un adecuado control. En operación, el intercambiador está sujeto al desgaste, ocasionado por sustancias de arrastre presente en los fluido e incrustaciones debido al almacenamiento de las sustancias que son transportadas por el fluido, por ejemplo, los gases que se separan del agua durante el calentamiento o por el fenómeno de contaminación del agua generando depósitos principalmente de sílice y lodos [20]. Además de la obstrucción y el desgaste, el intercambiador en la operación presenta perturbaciones, disturbios en el proceso, cambios intempestivos de las variables que degradan la operación del intercambiador y generan perdida de eficiencia en la operación de este, haciendo más relevante el control de todo el proceso de intercambio térmico.

Para realizar el control del intercambiador es importante conocer los elementos que componen el proceso de intercambio térmico. La Fig. 3 hace referencia a un intercambiador de calor [21] al que le entran dos fluidos, que corresponden al "producto" o fluido que se pretende calentar y el "fluido calor portador", fluido que va a calentar al producto.

El caudal del producto de entrada, $Q_{P}(t)$, es medido mediante un transmisor de flujo FIT 002, este fluido pasa a través de la válvula de control FCV 001, a la salida del intercambiador pasa por el caudalímetro FIT 005. La temperatura del producto es medida tanto a la entrada, $T_{E P}(t)$, como a la salida del intercambiador; en la entrada mediante los instrumentos termómetro TI 001, sensor TE 001 y el transmisor indicador de temperatura TIT 001, y en la salida a través del termómetro TI 003, el sensor TE 003 y el transmisor indicador de temperatura TIT 003.

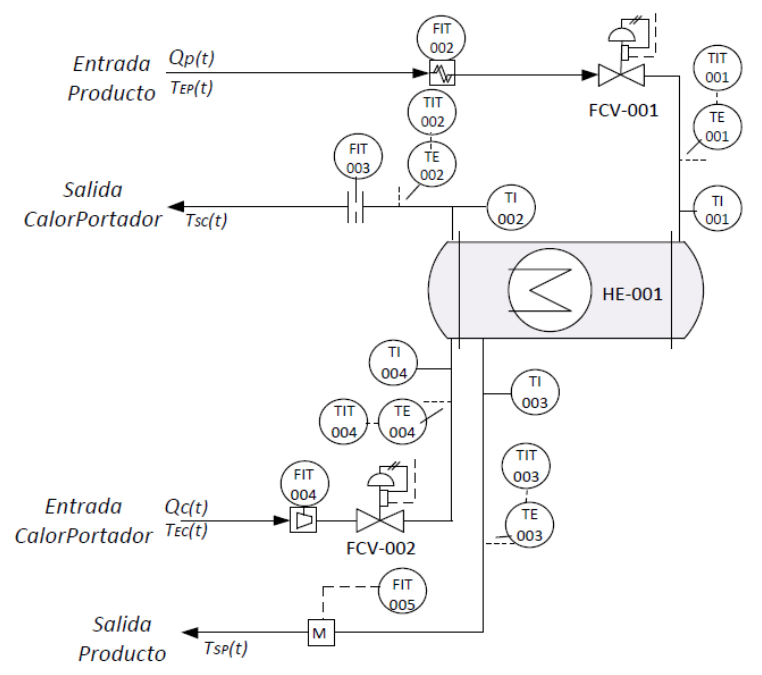

Fig. 3. Diagrama P\&ID intercambiador de calor [21]

El caudal de entrada $Q_{C}(t)$, fluido calor portador, es medido por el caudalímetro FIT 004, flujo que también pasa por la válvula de control FCV 002 y a la salida del intercambiador es medido por el transmisor de flujo FIT 005. La temperatura de entrada $T_{E C}(t)$ del fluido calor portador es medida mediante los instrumentos termómetro TI 004, sensor TE004 y el transmisor indicador de temperatura TIT004 y la temperatura de salida es medida por los instrumentos: termómetro TI 002, sensor TE002 y el transmisor indicador de temperatura TIT002.

Dentro de los factores para tener en cuenta en el control de los intercambiadores de calor se destacan la presión del vapor o del fluido de alimentación, las fluctuaciones en el caudal del producto, las variaciones en la temperatura del producto, calor específico, retardos del proceso. Considerando estos factores, es posible controlar: la temperatura en la línea de fluido calor portador, la temperatura en la línea de producto, temperatura en la línea de fluido energético con control de nivel de producto, control de temperatura mediante regulación primaria de presión y secundaria de flujo energético, control anticipativo (FeedForward) para la compensación de perturbaciones en un intercambiador de calor. En un adecuado sistema de control se espera que el proceso funcione en condiciones estables de operación independiente de perturbaciones y desajustes, que responda de manera oportuna, continua y que trabaje bien ante diferentes cambios o condiciones de operación del proceso.

\section{ESTRATEGIAS DE CONTROL}

En general, el objetivo de los sistemas de control es cumplir con las especificaciones de desempeño dadas para el 
proceso, definiendo valores o rangos considerados como aceptables para el comportamiento deseado. Típicamente, existen dos problemas de control [22]: seguimiento de la referencia y rechazo de disturbios. Sin embargo, en muchos procesos industriales el seguimiento de referencia es menos crítico que el rechazo de disturbio, debido a que los cambios de la referencia pueden darse únicamente cuando cambia la producción. La robustez e incertidumbre del modelo y sensibilidad al ruido de medición son objetivos secundarios de control. Entre las estrategias empleadas para realizar la transferencia calor y controlar la temperatura del producto, encontramos: Control realimentado (FeedBack), control anticipativo (FeedForward) [11], [23], para el rechazo de perturbaciones; control cascada [21] y el control por derivación para aplicaciones específicas.

\section{A. Control realimentado - FeedBack}

El objetivo del sistema es controlar la temperatura de salida del producto. El controlador cambia su salida en función al error entre la temperatura de referencia y la temperatura de salida del producto $T_{S P}(t)$. La principal ventaja de esta estrategia radica en su simplicidad de implementación y la capacidad de compensar cualquier tipo de perturbación del sistema [24]. En la Fig. 4 puede verse el esquema de control realimentado actuando directamente sobre la válvula de calor portador.

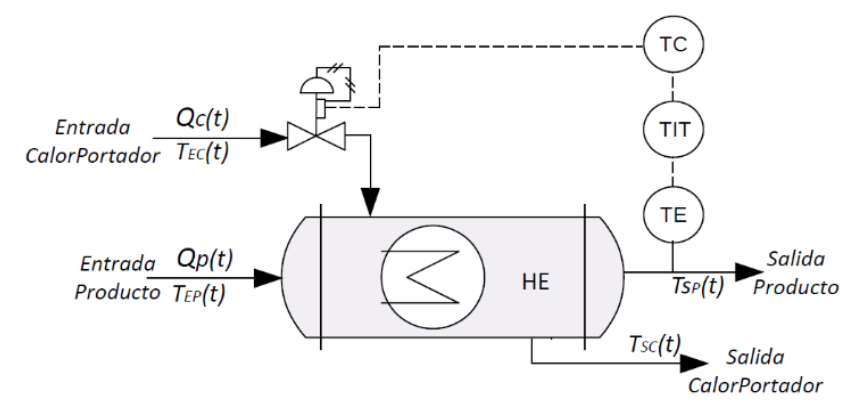

Fig. 4. Lazo de control realimentado (FeedBack) para el control de temperatura de salida del producto en un intercambiador de calor.

\section{B. Control anticipativo - FeedForward.}

Aunque el control realimentado es el más usado a nivel industrial, su desventaja radica en que la compensación de los disturbios no se realiza hasta que la variable controlada $T_{S P}(t)$ se desvíe del punto de operación, es decir, la perturbación debe propagarse en todo el proceso antes de ser compensada. $\mathrm{Si}$ se requiere recuperar el punto de operación rápidamente, se puede optar por la implementación de control anticipativo, de la Fig. 5, justificando la inversión en instrumentación adicional.

El control anticipativo se combina con el control realimentado, donde el primero compensa la variable controlada antes de que se desvié midiendo directamente las perturbaciones; y el segundo mantiene el punto de operación de la temperatura de salida. Las perturbaciones medibles en el proceso son: temperatura de entrada de producto $T_{E P}(t)$ y el flujo del producto $Q_{P}(t)$ manteniendo como la variable manipulada el flujo del fluido CalorPortador $Q_{C}(t)$.

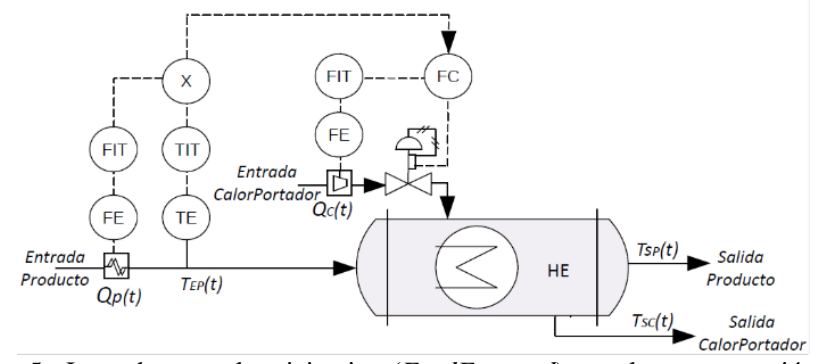

Fig. 5. Lazo de control anticipativo (FeedForward) para la compensación de perturbaciones en un intercambiador de calor.

\section{Control Cascada}

En el esquema de control cascada, Fig. 6, la salida del controlador de temperatura es la referencia del controlador interno de flujo o vapor basado en un lazo de realimentación simple [24]. Esta estrategia elimina los efectos de disturbios internos de entrada o salida del proceso, corrige las alinealidades e incertidumbres del lazo interno.

Para el caso de un controlador externo de temperatura y un controlador interno de flujo (ver Fig. 6), las variaciones del flujo CalorPortador son corregidas inmediatamente, por el controlador de flujo interno y el controlador de temperatura externo se encarga de compensar las variaciones de temperatura por otras causas. El significado físico de la señal del controlador de temperatura sería el flujo CalorPortador que se requiere para mantener la temperatura en el punto de operación. Hay que notar que el lazo de control interno debe ser mucho más rápido que el externo, cancelando las variaciones de flujo antes de que afecte la temperatura de salida del producto.

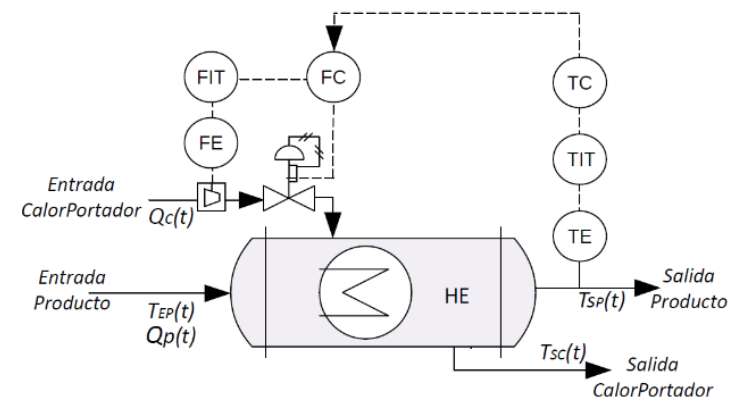

Fig. 6. Esquema de control cascada de un intercambiador de calor.

\section{Control Integrado}

La Fig. 7 muestra un control integrado de realimentación, anticipativo y cascada [15], que brinda un mayor grado de libertad en el ajuste de las especificaciones de diseño del sistema de control, por ejemplo:

- Rechazo de perturbaciones (Control anticipativo).

- Minimizar variación de flujo del fluido CalorPortador (Control cascada).

- Seguimiento de la referencia (control realimentado).

La integración de estas tres estrategias permite la optimización del control de temperatura del intercambiador de calor para minimizar la variación del proceso, maximizar la calidad del producto y garantizar la eficiencia energética del proceso. 


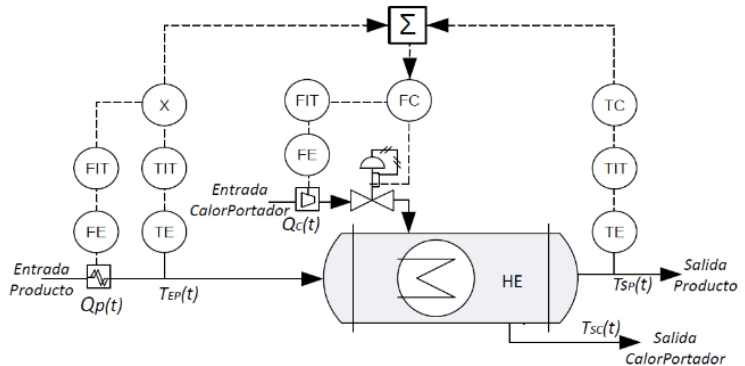

Fig. 7. Estrategia de control cascada más un control anticipativo para un intercambiador de calor [15].

\section{E. Control por derivación}

Si el intercambiador responde lentamente, la regulación de la temperatura se realiza con un controlador actuando sobre una válvula de tres vías para derivar el flujo del intercambiador. En [15] y [25] se muestra dos variaciones para este esquema de control: derivación en la línea CalorPortador y derivación en la línea del producto. Este esquema logra una respuesta más rápida frente a lazos de control con válvula de dos vías, debido a que se afecta casi inmediatamente la temperatura de salida al evitar que el flujo CalorPortador o producto atraviese el intercambiador. Hay que tener en cuenta que la derivación del producto no se puede realizar en aquellos procesos donde se requiera que todo el producto aumente o disminuya su temperatura, por ejemplo: procesos de pasteurización y esterilización.

\section{MODELO DEL INTERCAMBIADOR DE CALOR PARA EL CONTROL}

En el control de procesos, la identificación del sistema permite determinar, con suficiente exactitud para fines de control, la estructura y parámetros de un modelo matemático que reproduzca las variables de salida del proceso ante un conjunto de señales de entrada en el sistema real. Estos modelos matemáticos son usados para describir o entender la realidad de procesos químicos, mecánicos, eléctricos y biológicos.

En forma general existen tres metodologías de identificación de procesos [26]: la primera metodología, identificación analítica, hace uso de relaciones establecidas, por ejemplo leyes físicas o químicas, que se pueden aplicar al sistema; la segunda metodología, identificación noparamétrica, se basa en aplicar señales especiales de prueba al sistema para luego ajustar un modelo matemático; y tercera, la identificación paramétrica [27], que permite caracterizar al sistema con un mayor grado de ajuste ya que incorpora modelos de perturbación en su estructura.

\section{A. Identificación analítica}

El desarrollo del modelo se basa en las relaciones fisicoquímicas del proceso como balances de energía y masa [26]; obteniendo un modelo complejo y no lineal en la mayoría de los casos, requiriendo de una gran experiencia y conocimiento del proceso para su desarrollo.

El modelo matemático de un intercambiador de calor es escrito en términos de ecuaciones diferenciales parciales (PDEs) en [28] y [29]. La solución de las ecuaciones PDEs puede ser complicado, por lo tanto, un modelo de parámetros concentrados es recomendable cuando el propósito es el control del intercambiador, tal como se presenta en [30] y [31]. El modelo se obtiene dividiendo el largo de intercambiador en $\mathrm{N}$ regiones de igual tamaño y se asume que en cada región la temperatura es uniforme. El número de regiones depende de la precisión que se quiere obtener con el modelo. Para cada región se realiza un balance energético. Las ecuaciones (1) y (2) describen el balance energético en cada una de las regiones:

\section{$C_{p 1 .} \rho_{1 .} V_{1} \frac{d T_{1 a v g}}{d t}=C_{p 1 .} \rho_{1 .} Q_{c^{*}}\left(T_{e c}-T_{s c}\right)-U A\left(T_{1 \text { avg }}-T_{2 a v g}\right)$}

$C_{p 2 .} \rho_{2 .} V_{2} \frac{d T_{2 a v g}}{d t}=C_{p 2 .} \rho_{2} Q_{p} \cdot\left(T_{e p}-T_{s p}\right)+U A\left(T_{1 a v g}-\right.$
$\left.T_{2 \text { avg }}\right)$

(2)

Donde $T_{1 a v g}=\frac{T_{e c}+T_{s c}}{2}$ y $T_{2 a v g}=\frac{T_{e p}+T_{s p}}{2}$ son las temperaturas promedio. $C_{p 1}, C_{p 2}$ los coeficientes de calor especifico de cada fluido $\left[J /\left(K_{g}{ }^{\circ} \mathrm{C}\right)\right] ; Q_{c}, Q_{p}$ son los flujos volumétricos de los fluidos $\left[\mathrm{m}^{3} / \mathrm{h}\right] ; V_{1}, V_{2}$ volumen de cada región $\left[\mathrm{m}^{3}\right] ; \rho_{1}$, $\rho_{2}$ son las densidades de los fluidos $\left[K_{g} / \mathrm{m}^{3}\right] ; U$ coeficiente del intercambiador $\left[\mathrm{J} / \mathrm{s} \cdot \mathrm{m}^{2}{ }^{\circ}{ }^{\circ} \mathrm{C}\right] ; A$ área de contacto $\left[\mathrm{m}^{2}\right]$ y $t$ es el tiempo [s]. El modelo matemático definido con las ecuaciones (1) y (2) tiene la habilidad de aproximar el comportamiento transitorio de diferentes tipos de intercambiadores, tal como se muestra en [30].

\section{B. Identificación experimental}

Obtener el modelo matemático mediante la identificación analítica requiere de una gran cantidad de tiempo de ingeniería, sin mencionar las dificultades en el modelado ocasionadas por las dinámicas complejas y desconocidas del intercambiador. La alternativa a este problema es implementar un método experimental para realizar la identificación del sistema. Esta identificación realiza la aproximación de un modelo matemático a la respuesta, asignando valores numéricos a los parámetros del modelo mediante la observación de datos experimentales [32]. De acuerdo con [33], los métodos experimentales se pueden dividir en dos categorías: paramétricos y no paramétricos.

\section{1) Métodos no paramétricos}

Estos métodos de identificación se caracterizan por ser usados cuando las dinámicas del sistema no pueden ser expresada por un número finitos de parámetros y al ser aplicados no entregan valores a los parámetros del modelo sino curvas o funciones. Entre las señales de pruebas experimentales utilizadas para la identificación se encuentran las de tipo escalón y las secuencias binarias pseudoaleatorias (PRBS) [26]; en algunos casos se emplean también señales sinusoidales para determinar la respuesta en frecuencia del sistema.

La limitación de esta técnica radica en que los modelos obtenidos son completamente determinísticos, es decir no consideran modelos de perturbación, no apropiado para el desarrollo de estrategias avanzadas de control. Aunque estas técnicas son sensibles al ruido y de baja precisión, son empleadas a nivel industrial debido a la fácil implementación. 
Sin embargo, perturbar mediante una señal de prueba a un proceso industrial en operación puede ocasionar modos no deseados, alterando la producción, por tal razón las empresas optan en no realizar la identificación en línea.

Los métodos implementados a nivel industrial se basan en la curva de reacción del proceso, como el método de la tangente de Zieglers-Nichols que aproxima la dinámica del sistema a un primer orden mediante una recta tangente al punto de inflexión de la curva de reacción; en [7] se emplea para el diseño de un control difuso. En [33], en lugar del punto de inflexión se emplea el valor del $63 \%$ de la salida. Otro método de identificación que aproxima la dinámica a un primer orden se muestra en [34] (método de Smith).

Para aproximaciones del intercambiador de calor a un sistema de segundo orden, el método de polos múltiples de Strej con o sin tiempo muerto es utilizado en [9], donde se emplea una multiplicidad de polo de dos y en [8], y en [13] con multiplicidad tres para el diseño de un controlador $\mathrm{H}_{2}$ y un $\mathrm{H} \infty$.

En [35], se presenta una comparación de técnicas de identificación de modelos matemáticos para sistemas de primer y segundo orden. Algunas de estas técnicas como: método de tres puntos (Jahanmiry y Fallanfi, Startk), Métodos de áreas característica de Nishikawa, Vitecková; no se han implementado en ambientes industriales para la identificación del modelo de intercambiadores de calor. Sin embargo, estas técnicas pueden emplearse sin limitaciones en la identificación del modelo.

\section{2) Métodos paramétricos}

La adaptación de la teoría de mínimos cuadrados en la identificación se basa en aceptar como cierto de que el proceso puede ser representado por un modelo de estructura fija, generalmente una ecuación lineal en diferencias, lo que implica que dicho modelo tiene naturaleza discreta [26]. Estos algoritmos pueden emplearse tanto fuera de línea (no recursiva) para determinar un modelo invariante con el tiempo o en línea (recursiva) para estimar parámetros variables con el tiempo.

En muchas investigaciones se han usado métodos paramétricos para obtener el modelo matemático de intercambiadores de calor, donde se aplican señales seudo aleatorias (PRBS) como señales de prueba. Los resultados muestran que es posible describir el comportamiento del intercambiador con estos modelos. En [16], se aplica el modelo ARX a cuatro intercambiadores de calor de diferente tipo. En [36] se hace uso de un modelo ARMAX para obtener el modelo del intercambiador y luego hacer uso del modelo para diseñar dos controladores PID's diferentes.

\section{Algoritmos DE CONTROL Y SINTONÍA}

A nivel industrial se emplean pocos controladores, Ver Fig. 1, sin embargo, existen una gran variedad de controladores avanzados aplicados a nivel académico siendo en su mayoría simulaciones. El controlador PID sintonizado con el método de curva de reacción de Ziegler-Nichols (ZN) [24] es el método tradicional en la industria gracias a su simplicidad en las reglas de sintonía basadas en el decaimiento de 1/4 del error; este criterio de sintonía puede ocasionar un pobre amortiguamiento y poca robustez debido a las altas ganancias del controlador. En [10],[12],[23],[37] se realiza un control basado con estas reglas de sintonía para intercambiadores coraza-tubo simulados, en [11] se realiza una simulación adicionando un control anticipativo para el flujo de entrada, el cual disminuye el tiempo de establecimiento y el sobrepaso. En [21] se realiza un control PI Ziegler-Nichols versus la herramienta PIDTuner de Matlab ${ }^{\circledR}$ en un intercambiador de laboratorio de nivel industrial.

Ziegler-Nichols es un método basado en tablas, de ahí radica su gran acogida en la industria, pero debido a los grandes sobrepasos que puede ocasionar, algunos autores han modificado los valores de sintonía del controlador PID [25]. En [8],[13],[37] se aplica en simulación las reglas de CohenCoon obteniendo respuestas similares al ZN; en [37] se hace uso de las reglas CHR (Chien-Hrones-Reswick), la cual tiene una variación para realizar un ajuste para seguimiento o regulación, este controlador presenta menores sobrepasos. Por último, en [8], se implementa un controlador Strej para un modelo de intercambiador de polos múltiples.

Ziegle-Nichols tiene una segunda metodología de sintonía, la cual implica llevar al sistema al borde de la inestabilidad para determinar la ganancia y frecuencia critica. Este proceso puede no ser seguro para el intercambiador de calor, por lo cual se emplea el método de oscilación por relé [38]; en [23] se simula este controlador y en [36] se implementa en un control real obteniendo la ganancia y frecuencia critica sin necesidad de llevar al sistema a un punto de operación no segura.

Como se mencionó en la sección IV, el modelo del intercambiador de calor posee una dinámica de tiempo muerto, por lo cual un controlador lineal como el PID no puede compensar efectivamente este efecto; por lo que se requiere el uso de controles predictivos. El controlador Smith es considerado como el primer algoritmo predictivo de control [39] al contener en su estructura el modelo matemático del sistema. En [11], el predictor Smith prueba que puede compensar los efectos del tiempo muerto solamente si se conoce el modelo exacto del intercambiador de calor; además, se implementa en conjunto con una estrategia FeedForward con lo cual disminuye las fluctuaciones de temperatura debido al cambio del flujo de entrada. En [40], se realiza la comparación de un predictor Smith con un PI por ZieglerNichols y un PID Tyreus-Luyben. El control por modelo interno (IMC) [41], es una estructura que hace uso de un modelo lineal del proceso en paralelo con el proceso real, para la compensación del tiempo muerto; mediante el ajuste de un solo parámetro. En [8],[23] se prueba en simulación que el control IMC limita los efectos del error causados por la incertidumbre en el modelo; en [36] se demuestra que el IMC responde mejor que el controlador por relé de Aström.

En [37], presentan controladores óptimos en un intercambiador de calor simulado basados en los criterios de la integral (Ver sección VI) de López [42]. El criterio de desempeño consiste en la minimización de algún criterio integral y la respuesta sólo es óptima para este criterio. El articulo [37] presenta dos tipos de sintonía dependiendo de los objetivos de control: para seguimiento o regulación. Y 
demuestra mejorar la respuesta transitoria del intercambiador de calor comparado contra un $\mathrm{ZN}$ y Cohen-Coon.

En [5] se emplea en un tren de intercambiadores de coraza tubo de cuatro etapas para un proceso de condensado de vapor, dos algoritmos de control no lineal: Generic Model Control GMC y Process model based control - PMBC [43] para obtener un rango de operación más amplio que el controlador PI clásico (modelo linealizado en el punto de operación) y contrarrestar las no linealidades del proceso. La ley de control se determina mediante un modelo y respuesta de proceso deseada, es decir son controladores basados en modelos: predictivo y adaptativo. Los controladores permiten la sintonización en línea, pudiendo ser posible utilizar los procedimientos heurísticos de un PI clásico. El GMC permite manejar las no linealidades del intercambiador, pero el PMBC compensa alinealidades y tiempos muertos variantes mediante un solo parámetro de sintonía. La dificultad de este algoritmo radica en la sintonía, la cual es considerada un arte, debido a que la especificación del modelo afecta a la ley de control resultante [44].

En cuento a las técnicas de control robusto tenemos el controlador $\mathrm{H}_{2}$ en [13], que garantiza la estabilidad interna del sistema y muestra una respuesta amortiguada y el controlador $H \infty$ [12],[13] que garantiza estabilidad y desempeño (sub)óptimo. Ambos controladores garantizan margen de módulo, dinámica de seguimiento rápida y rechazo de ruido dependiendo de las restricciones físicas del sistema. Sin embargo, el controlador $\mathrm{H} \infty$ es un controlador de alto orden difícil de implementar y requiere un gran conocimiento del sistema para selección de las funciones de peso. En [45] se presenta dos controladores robustos diseñados por el criterio de frontera de estabilidad y el teorema de pequeña ganancia.

Los controladores inteligentes toman en cuenta la lógica del proceso, optimización, probabilidad, percepción, razonamiento humano, toma de decisiones y aprendizaje; su objetivo es integrar agentes inteligentes en los sistemas de control [46]. En [10] se hace uso de algoritmos genéticos para resolver el problema de optimización de los parámetros del controlador PID para un amplio rango de puntos de operación del intercambiador de calor. En [8] un controlador predictivo basado en modelo de red neuronal es usado para predecir el comportamiento futuro del intercambiador de calor y minimizar un criterio de desempeño. En [7] se realiza la comparación entre un controlador $\mathrm{ZN}$, un controlador difuso lineal y no lineal, mostrando que el difuso no lineal presenta un mejor desempeño de regulación y amortiguamiento de la respuesta del intercambiador. En [47], se compara un controlador difuso convencional contra un PI difuso híbrido en un intercambiador coraza tubo de laboratorio y empleando el software Matlab para implementar el controlador, el controlador híbrido demuestra tener un mejor desempeño evaluado mediante los criterios de IAE e ISE.

\section{VI. ÍNDICES DE EVALUACIÓN DE DESEMPEÑO}

Los requerimientos de control de un intercambiador de calor se definen por el proceso, ya que es este el que indica el punto de operación de temperatura, presiones y flujos de entrada. Por lo tanto, no existen índices estandarizados y valores que se consideren únicos para el intercambiador de calor. La evaluación de los lazos de control en la Industrial del Valle en la mayoría de los casos depende de la experiencia del operario y del ingeniero de control, quienes seleccionan los índices de evaluación de desempeño y el rango de valores que se consideran aceptables. El tiempo de estabilización "ts" y el sobrepaso máximo de la respuesta "Mp", son los índices de mayor uso en la literatura académica, en su mayoría en simulación del intercambiador 74\%; sin embargo, a nivel industrial no se consideran relevante, se encontró que la variación de la temperatura del punto de operación (error) es el índice de evaluación empleado. Las variaciones de flujo y presión indican fallas en el proceso. La mayoría de los índices de evaluación que se encuentran en la literatura sobre lazos de control de temperatura en intercambiadores de calor pertenecen al dominio del tiempo.

- Tiempo de establecimiento - ts: tiempo requerido para que la temperatura de salida del producto alcance y permanezca dentro de una banda determinada $(\delta)$ alrededor del valor final (usualmente $\delta= \pm 5 \%$ o $\pm 2 \%$ del cambio de la salida). Este índice de desempeño determina la velocidad de respuesta. Es usado en [7], [10]-[12], [23], [37], [48].

- Sobrepaso - Mp: máximo valor de temperatura de salida del producto que excede el valor final después de una alteración del sistema. Es un indicador de dinámicas subamortiguadas y/ó presencia de un cero en el semiplano complejo izquierdo. Junto al tiempo de establecimiento es usado en [7],[10]-[12], [23], [37], [48].

En general, el sistema de control del intercambiador de calor busca minimizar el error de la variable de proceso, en función del tiempo transcurrido, después de haberse producido una alteración en el sistema (Cambio de la temperatura de entrada del producto, cambio del flujo del producto). La magnitud del error y el tiempo durante el cual existe, contribuyen a la definición de control óptimo [49]. Los criterios de las integrales se definen por una función de costo (ver Ecuación 3), donde F es función del error y el tiempo. El criterio de comportamiento $\varphi$ será cero solamente si el error es cero durante todo el tiempo, situación imposible en presencia de perturbaciones en el sistema. Por lo tanto, $\varphi$ no será nulo, pero cuanto más pequeño sea su valor mejor será el comportamiento del sistema.

$$
\varphi=\int_{0}^{\infty} F|e(t), t| d t
$$

Los índices de desempeño basado en criterios de integrales "optimas" encontrados en la literatura son: IAE (Integral of the Absolute Value of the error) [8],[13],[23],[45],[48],[50], este criterio es sensible a pequeños errores, es un indicador de un pobre amortiguamiento del lazo cerrado, la desventaja de este criterio es que su evaluación requiere una gran cantidad de cálculo o simulación del proceso; ISE (Integral of the Square of the error)[8],[23],[36],[48],[50], es relativamente insensible a pequeños errores, pero los grandes errores contribuyen fuertemente al valor de la integral. ITAE (Integral of the time-Weighted Absolute of the Error) [26],[36], es insensible a errores iniciales y penaliza fuertemente los errores que permanecen a lo largo del tiempo, indicador de tiempo de respuesta cortos y sobrepasos; por último, ITSE (Integral of 


\section{CONCLUSIONES}

El estudio del control del intercambiador de calor no es un tema nuevo en la ingeniería de control, se encuentra que varios autores proponen técnicas de control clásicas, robustas e inteligentes, entre otras; sin embargo, en su mayoría estas técnicas son aplicadas a procesos simulados despreciando las no linealidades ocasionadas por las válvulas, bombas e instrumentación presenten en un proceso industrial real. Un gran aporte a la academia y a ingeniería seria comprobar dichas técnicas de control en plantas industriales o plantas pilotos que impliquen los retos reales de implementación.

A partir de visitas realizadas a empresas en la región del Valle del Cauca se pudo evidenciar que aún se presenta un gran desconocimiento por los técnicos de las empresas en metodologías de sintonía de controladores PID detectándose aun problemas comunes en los controladores PID tales como: alta variabilidad, bajo rendimiento, poca eficiencia en los lazos de control, lo cual muestra que abordar temas de la ingeniería en capacitación y transferencia son requeridos buscando mejorar la competitividad y productividad de las empresas.

De igual forma se pudo determinar que técnicas avanzadas como controladores inteligentes y robustos no son aún usados en la industria, principalmente debido al desconocimiento en la sintonía, implementación y mantenimiento de estos controles por parte de ingeniería y operación. Implementar, validar y documentar los métodos no usados en la industria en plantas reales es otro reto para la ingeniería y la academia de la región del Valle del Cauca.

De acuerdo con el análisis realizado, mejorar el comportamiento en automático de los intercambiadores de calor implican varios componentes fundamentales: conocer el modelo proceso, seleccionar la estrategia más adecuada para dicho modelo, ajustar y sintonizar el algoritmo de control y aplicar criterios de evaluación de desempeño. Como ejemplo, la aplicación de estos componentes al tratamiento térmico llevaría a la obtención de productos inocuos, de mejor calidad y con mayor tiempo de conservación, en el caso de los alimentos.

\section{AGRADECIMIENTOS}

Los autores agradecen al Ingeniero Alfredo Espinosa de Alival, a los ingenieros Darío Torres y Alejandro Sayur de Propal, ingeniero Raúl Andrés Herrera (Professional Specialist in Automation and Process Control) y al ingeniero Jhosen Calderón (PMP, Senior Project Engineer) de Ingredion, Ingeniero Carlos Andres Sanchez (Planner de Mantenimiento E\&I) de Smurfit Kappa, ingeniero Iber Rivera Mariño (Coordinador - Automatización / instrumentación y control) del Ingenio Providencia S.A., ingeniero Jesús Alberto González MsC. docente titular de la Universidad del Valle de las asignaturas "Control de procesos" e "Instrumentación Industrial" por sus contribuciones en el desarrollo de la encuesta e información brindada de sus procesos y sistemas de control.
[1] J. Jilek, (2016, may). "Heat exchangers in europe,". [Online]. Available: www.cbi.eu/market-information

[2] M. Ruel, "The conductor directs this orchestra. Shift from pneumatic instrumentation to ultramodern equipment no panacea." InTech Magazine, 2003.

[3] Bernardo Soares Torres, F'abio Barros de Carvalho, "Perfomance assessment of control loops - case studies," IFAC 2006.

[4] D. V. Mullick S, "Consider integrated plant design and engineering," Hydrocarbon Processing, pp. 81 - 85, Dec. 2007.

[5] P. R. Raul, H. Srinivasan, S. Kulkarni, M. Shokrian, G. Shrivastava, and R. R. Rhinehart, "Comparison of model-based and conventional controllers on a pilot-scale heat exchanger," ISA Transactions, vol. 52, no. 3, pp. $391-405,2013$.

[6] F. Kuo, Benajmin. Golnaraghi, Ingenieria de Control Moderna, 9th ed. John Willey \& Sons, Inc, 2010.

[7] A. V. Duka and S. E. Oltean, "Fuzzy control of a heat exchanger," Proceedings of 2012 IEEE International Conference on Automation, Quality and Testing, Robotics, pp. 135-139, May 2012.

[8] A. Vasickaninova and M. Bakosova, "Control of a heat exchanger using neural network predictive controller combined with auxiliary fuzzy controller," Applied Thermal Engineering, vol. 89, pp. 1046 - 1053, 2015.

[9] A. Vasickaninova, M. Bakosova, A. Meszaros, and J. Oravec, "Fuzzy controller design for a heat exchanger," 2015 IEEE 19th International Conference on Intelligent Engineering Systems (INES), no. 1, pp. 225230, 2015.

[10] Y. L. G. H. Jianhua Zhang, Wenfang Zhang, "Controller design for a heat exchanger in waste heat utilizing systems," Advances in Swarm Intelligence. Second International, ICSI 2011, Chongqing China. Proceedings, Part II. Springer., 2011.

[11] A.-V. DUKA, M. DULA U U, and S.-E. OLTEAN, "Robust control of a heat exchanger using a smith predictor," Interdisciplinarity in Engineering International Conference "Petru Maior" University of Tirgu Mures, 2012.

[12] M. Dulau, S. Oltean, and A. Gligor, "Conventional control vs. robust control on heat-exchangers," Procedia Technology, vol. 19, pp. 534 $540,2015$.

[13] A. Vasi`ckaninov'a and M. Bako`sov'a, "Application of H2 and H1 Approaches to the Robust Controller Design for a Heat Exchanger," Chemical Engineering Transactions, 112013.

[14] K. Ogata, Ingeniería de Control Moderna, 5th ed. Pearson Education. S.A., 2010.

[15] C. Antonio, Instrumentation Industrial, 8th ed. Alfaomega Grupo Editoe, S.A. de C.V., México, 2010.

[16] Tatang Mulyana, Farah Najaa Suhaimi, "Arx model of four types heat exchanger identification," Jan. 2011, sin publicar.

[17] S. P. S" oderstr" om. Torsten, System Identification, 1st ed. Prentice Hall International, 1989.

[18] R. Serth, Process HEat Transfer Principles and Applications, 1st ed. Elsevier, 2007, vol. 1.

[19] D. G. Mendisaval, Intercambiadores de calor: Tipos Generales y Aplicaciones., mar 2002, ch. 1, p. 3.

[20] Manual de Intercambiador de calor. Instrucciones y Mantenimiento, 2010.

[21] Álvarez D. Juan Gonzalo, Moreno S. Jorge Enrique, "Diseño e implementación de un sistema de control cascada en la planta de intercambio térmico - pit000," Informado Técnico (Colombia) 81(1) Enero - junio 2017, pp. 32-43, 2017.

[22] K. J. A^ stro"m and T. Ha"gglund, PID controllers: Theory, Design, and Tuning, 2nd ed. Instrument Society of America, Research Triangle Park, NC, 1995, vol. 10.

[23] S. PADHEE, "Controller design for temperature control of heat exchanger system: Simulation studies," WSEAS TRANSACTIONS on SYSTEMS and CONTROL, vol. 9, 2014.

[24] A. B. Smith, Carlos A. Corripio, Principles and Practice of Automatic Process Control, 2nd ed. John Willey \& Sons, Inc, 1997.

[25] B. W. Bequette, Process Control. Modeling, Design and Simulation. Prentice Hall International Series in the Physical and Chemical Engineering Sciences, 2003, vol. 1.

[26] M. M. I. Alberto Aguado Behar, Identificación y Control Adaptativo, 1st ed. Pretice Hall, S.A., 2003. 
[27] T. Mulyana, "Parametric and non-pametric identification of shell and tube heat exchanger mathematical model," Ph.D. dissertation, Faculty of Electrical and Electronic Engineering, Universiti Tun Hussein Onn Malaysia, 2014.

[28] A. Vasi`ckaninov'a, M. Bako`sov'a, A. M'esz'aros, and J. J. Kleme`s, "Neural network predictive control of a heat exchanger," Applied Thermal Engineering, vol. 31, no. 13, Aug. 2011.

[29] Vera-García a, J.R. García-Cascales, "A simplified model for shell-andtubes heat exchangers: Practical application," Applied Thermal Engineering, no. 30, pp. $1231-1241,2010$

[30] P. Laszczyk, "Simplified modeling of liquid-liquid heat exchangers for use in control systems," Applied Thermal Engineering, vol. 119, pp. 140-155, Jun. 2017.

[31] A. K. Alexander Michel, "Accurate low-order dynamic model of a compact plate heat exchanger," International Journal of Heat and Mass Transfer, no. 61, pp. 323 - 331, 2013.

[32] H. Werner, Control Systemes Theory and Design. Technische Universit"at Hamburg, oct 2012, ch. 7, p.290.

[33] T. Mulyana, "A nonparametric system identification based on transient analysis with plant process of heat exchanger as study case," International Journal of Innovation in Mechanical Engineering and Advanced Materials, vol. 1, no. 1, pp. 19-26, 2015.

[34] D. Hanafi, M. N. M. Than, A. A. A. Emhemed, T. Mulyana, A. M. Zaid, and A. H. Johari, "Heat exchanger's shell and tube modeling for intelligent control design," 2011 IEEE 3rd International Conference on Communication Software and Networks, pp. 37-41, May 2011.

[35] V. M. Alfaro, "Identificación de procesos sobre amortiguados utilizando técnicas de lazo abierto," Ingeniería. Revista de la Universi*dad de Costa Rica, vol. 11, no. 1,2, 2001.

[36] A. Sahoo, T. Radhakrishnan, and C. S. Rao, "Modeling and control of a real time shell and tube heat exchanger," Resource-Efficient Technologies, vol. 3, no. 1, pp. 124 - 132, 2017.

[37] S. B. Prusty, S. Padhee, U. C. Pati, and K. K. Mahapatra, "Comparative performance analysis of various tuning methods in the design of pid controller," Michael Faraday IET International Summit: MFIIS-2015, pp. 43-48, Sept 2015.

[38] K. J. A^ stro"m and Ha"gglund, "Automatic tuning of simple regulator with specifications on phase and amplitud margins," 1984 International Federation of Automatic Control, vol. 20, no. 5, pp. 645-651, 1984.

[39] V. Bob'al, M. Kubal'c'ik, and P. Dost'al, "Identification and self-tuning predictive control of heat exchanger," 2013 International Conference on Process Control (PC), pp. 219-224, June 2013

[40] G. M. Sarabeevi, "Dead time compensation in shell and tube heat exchanger system using smith predictor," International Journal of Science and Research (IJSR), no. 5, pp. 1853 - 1885, 2016.

[41] W. Tan, J. Liu, T. Chen, and H. J. Marquez, "Comparison of some wellknown pid tuning formulas," Computers \& Chemical Engineering, vol. 30, no. 9, pp. $1416-1423,2006$.

[42] V. M. Alfaro, "Método de sintonización de controladores pid que operan como reguladores," Ingeniería. Revista de la Universidad de Costa Rica, vol. 12, no. 1,2, 2002.

[43] H. Subawalla, V. P. Paruchuri, A. Gupta, H. G. Pandit, and R. R. Rhinehart, "Comparison of model-based and conventional control: a summary of experimental results," Industrial \& Engineering ChemistryResearch, vol. 35, no. 10, pp. 3547-3559, 1996.

[44] B. W. Bequette, "Nonlinear control of chemical processes: a review," Industrial \& Engineering Chemistry Research, vol. 30, no. 7, pp. 1391$1413,1991$.

[45] M. B. A. Vasi ckaninov'a, "Robust controller design for a heat exchanger," 2015 International Conference on Process Control (PC), 2015.

[46] A. Alam, R. P. Gupta "Performance analysis of intelligent controller for temperature of heat exchanger," International conference on Signal Processing Communication, Power and Embedded System (SCOPES), pp. $12-17,2016$.

[47] K. Kishore, G. Jalalu, K. Prasanti "Control of heat exchanger using hybrid fuzzy - pi," International Journal of Engineering Research and Applications (IJERA), pp. 1396 - 1400, 2013.

[48] S. Padhee, Y. B. Khare, and Y. Singh, "Internal model based pid control of shell and tube heat exchanger system," Students' Technology Symposium (TechSym), 2011 IEEE, pp. 297-302, Jan 2011.

[49] S. D. M. Smith Carlos. Corripio, Armando. Basurto, Control automático de procesos: teoría y práctica. Limusa, 1991.
[50] P. Chalupa, V. Bobál, M. kubalcic, J. Novac "Adaptive predictive control of through-flow heat exchanger," 18th Mediterranean Conference on Control \& Automation, 2010.

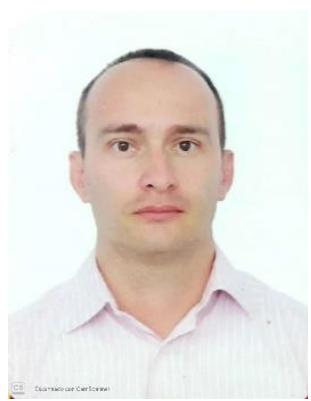

Juan Gonzalo Álvarez Diaz. Nació en Manizales, Caldas. Se graduó de la Universidad Nacional de Colombia como Ingeniero Electrónico. Obtuvo su título de maestría en Ingeniería de la Universidad del Valle, Cali, en 2011. Es instructor del área de instrumentación y automatización en el Servicio Nacional de Aprendizaje - SENA desde el 2008. Ha sido profesor hora catedra en la Universidad del Valle desde el 2011. Sus áreas de interés son la instrumentación, el control de procesos, Comunicaciones industriales y sistemas SCADA.

ORCID: https://orcid.org/0000-0002-8976-5290

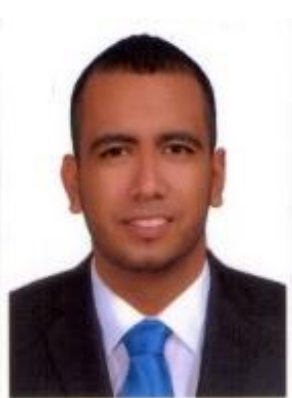

Juan Manuel Armero Viveros Nació en Cali, Valle del Cauca. Se graduó de la Universidad del Valle de Colombia como ingeniero Electrónico. Obtuvo su título de maestría en ingeniería de la Universidad del Valle, Colombia, en 2018. Ha sido instructor del área de automatización Industrial en el servicio nacional de Aprendizaje - SENA. Actualmente es ingeniero de instrumentación e infraestructura en CELSIA. Sus áreas de interés son el control de procesos, electrónica de potencia e instrumentación Geotécnica.

ORCID: https://orcid.org/0000-0002-0192-8973

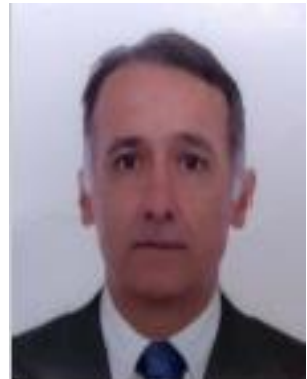

Carlos Andres Urrutia. Nació en Popayán, Cauca, Colombia. Se graduó de la Universidad del Cauca. Obtuvo título como Ingeniero en Electrónica con estudios en Telecomunicaciones. Soy Evaluador de Competencias Laborales en Instrumentación y Automatización, Sistemas de Energías Renovables en el Servicio Nacional de Aprendizaje SENA. Docente de la Institución Universitaria Antonio José Camacho desde el 2010. Áreas de interés instrumentación, control de procesos y sistemas de energías renovables. ORCID: https://orcid.org/0000-0002-7686-0387 